\title{
Limites e desafios para o acesso das mulheres privadas de liberdade e egressas do sistema prisional nas Redes de Atenção à Saúde
}

\author{
I ${ }^{1}$ Águida Luana Veriato Schultz, ${ }^{2}$ Renata Maria Dotta, ${ }^{3}$ Bárbara Sordi Stock, \\ ${ }^{4}$ Míriam Thais Guterres Dias I
}

Resumo: O trabalho analisa os limites e desafios para o acesso da populaçáo prisional feminina e egressa do sistema prisional nos serviços da Rede de Atenção à Saúde (RAS). Trata-se de estudo qualitativo, exploratório e descritivo realizado com dez trabalhadores de uma Equipe de Atenção Básica prisional de um presídio feminino na Regiáo Sul do Brasil. Realizaram-se sessōes de grupo focal e os dados foram analisados tematicamente, resultando em dois eixos: (a) Barreiras para inclusão das reclusas na RAS; e (b) Barreiras para inclusão das egressas na RAS. Apesar de a unidade básica de saúde prisional ser considerada a principal porta de entrada da população carcerária no sistema de saúde, ainda precisa ser organizada para tornar-se ordenadora do cuidado à saúde da mulher. As dificuldades para o acesso das egressas nos serviços de saúde extramuros contribuem para a descontinuidade do tratamento, haja vista a falta de acompanhamento desta populaçáo pelos componentes da RAS, especialmente pelos serviços de saúde mental. Faz-se necessário avançar na ampliaçáo e otimização de serviços mais equitativas e integrais, estimulando a integração na gestão de fluxos entre a atenção básica prisional e os demais dispositivos da RAS.

> Palavras-chave: prisões; Atenção Primária à Saúde; Redes de Atenção à Saúde; saúde mental; gênero e saúde.

\author{
${ }^{1}$ Instituto de Psicologia, \\ Universidade Federal do Rio Grande \\ do Sul. Porto Alegre-RS, Brasil \\ (aguida_schultz@hotmail.com). \\ ORCID: 0000-0002-4716-6783 \\ ${ }^{2}$ Faculdade de Direito, Fundação \\ Escola Superior do Ministério \\ Público. Porto Alegre-RS, Brasil \\ (renata.dotta@uol.com.br). \\ ORCID: 0000-0002-1830-1624 \\ ${ }^{3}$ Universidad Católica de \\ Temuco, Facultad de Ciencias \\ Jurídicas. Temuco, Chile \\ (barbarasordistock@gmail.com). \\ ORCID: 0000-0001-9148-0596 \\ ${ }^{4}$ Instituto de Psicologia, \\ Universidade Federal do Rio \\ Grande do Sul. Porto Alegre-RS, \\ Brasil (miriamtgdias@gmail.com) \\ ORCID: 0000-0002-3881-4961
}

Recebido em: 15/06/2020 Aprovado em: 26/07/2020 Revisado em: 10/09/2020 


\section{Introdução}

A atenção à saúde em ambientes de privação de liberdade é uma questão sanitária importante, embora, muitas vezes, negligenciada pelo poder público. O princípio da equidade em saúde é claramente compreendido pelos regramentos internacionais ao prever que a oferta de serviços de saúde nas prisóes seja equivalente aos fornecidos à comunidade em geral. Globalmente, a atenção à saúde da população privada de liberdade no sistema prisional é considerada um direito constitucional e uma necessidade de saúde pública (WHO, 2007).

Especificamente em relação à atenção à saúde, a Regra no 6 das Regras de Bangkok - regras das Naçôes Unidas para o tratamento de mulheres presas e medidas não privativas de liberdade para mulheres infratoras - estabelece que o exame médico da mulher presa deve ser capaz de determinar a necessidade de cuidados de saúde básicos e, também, a presença de doenças sexualmente transmissíveis, cuidados de saúde mental, histórico de saúde reprodutiva, dependência de drogas e violência de gênero (BRASIL, 2016). Trata-se do principal marco normativo internacional que aborda essa questão e que serve de complemento para as regras mínimas para o tratamento de reclusos, também conhecida como Regras de Mandela (UNODOC, 2015).

No Brasil, o direito à saúde da população privada de liberdade é regulado por alguns parâmetros normativos como: a Lei de Execução Penal (LEP), de 1984; a Constituição Federal de 1988; as Leis Orgânicas no 8.080 e 8.142, de 1990, que regulamentam o Sistema Único de Saúde (SUS); o Plano Nacional de Saúde no Sistema Penitenciário (PNSSP), de 2003; a Portaria no 4.279, de 2010, que estabelece diretrizes para a organização da Rede de Atenção à Saúde (RAS) no âmbito do SUS; o Decreto no 7.508, de 2011, que regulamenta a Lei no 8.080, para dispor sobre a organização do SUS, o planejamento da saúde, a assistência à saúde e a articulação interfederativa; a Política Nacional de Atenção Integral à Saúde das Pessoas Privadas de Liberdade no Sistema Prisional (PNAISP), de 2014; a Política Nacional de Atenção às Mulheres em Situação de Privação de Liberdade e Egressas do Sistema Prisional (PNAMP), de 2014; a nova Política Nacional da Atençâo Básica, aprovada pela Portaria no 2.436, de 2017, que reconhece a PNAISP enquanto um dispositivo do SUS, considerando os princípios da equidade, da universalidade e da integralidade do acesso às pessoas privadas de liberdade na RAS e a Portaria no 99, de 2020, que 
redefine o registro das Equipes de Atenção Primária e Saúde Mental no Cadastro Nacional de Estabelecimentos de Saúde (CNES) (SCHULTZ et al., 2017).

Sobretudo a partir da PNAISP, abriu-se a possibilidade de constituir equipes de atenção básica prisional (EABp) por meio do financiamento estabelecido pela Portaria GM/MS no 482/2014 do Ministério da Saúde (BRASIL, 2014a; BRASIL, 2014b). Coube às EABp a função de realizar a atenção primária à saúde nas instituiçôes prisionais, integrando esse serviço à Atenção Primária à Saúde (APS) municipal, que têm a obrigatoriedade de utilizar os sistemas de informação, de acesso à educação permanente e de acesso regulado às Redes de Atenção à Saúde (RAS). Estas se caracterizam pela formação de relaçóes horizontais entre os pontos de atenção, tendo como centro de comunicação a APS. Fundamentam-se na compreensão da APS como primeiro nível de atenção, enfatizando a função resolutiva dos cuidados primários sobre os problemas mais comuns de saúde. Têm como objetivo promover a integração sistêmica de ações e serviços de saúde com provisão de atenção contínua, integral, de qualidade, responsável e humanizada, bem como incrementar o desempenho do sistema de saúde, em termos de acesso, equidade, eficácia clínica e sanitária, e eficiência econômica (MENDES, 2015).

A organização de redes busca a superação do modelo tecnoassistencial centrado em serviços e procedimentos, apostando na organização da atenção integral e tendo como ação estratégica a implantação das Linhas de Cuidado. A Linha de Cuidado é a imagem pensada para expressar fluxos assistenciais seguros e garantidos ao usuário, no sentido de atender às suas necessidades de saúde nos diferentes momentos do ciclo vital, tendo como eixo norteador a busca pela integralidade e equidade. Ela desenha o itinerário que o usuário faz por dentro de uma RAS, incluindo açóes e serviços não necessariamente inseridos no sistema de saúde, mas que participam de alguma forma da rede de cuidados (FRANCO; MAGALHÁES, 2003).

O modelo de atenção proposto busca reorganizar o sistema nas práticas da gestão e coordenação do cuidado em saúde. O sentido atribuído à coordenação do cuidado exprime a ideia de "anything that bridges gaps" (preencher lacunas ou vazios assistenciais). Logo, coordenar significa estabelecer conexôes e alcançar o objetivo de prover/atender às necessidades e preferências dos usuários na oferta de cuidados, com elevado valor e qualidade. $\mathrm{O}$ ato de coordenar implica a organização deliberada de atividades que envolvem duas ou mais pessoas (incluindo o usuário do serviço/ 
sistema de saúde) e o manejo de recursos, de modo a produzir uma oferta adequada do cuidado necessário (McDONALD et al., 2014).

Naturalmente, para o planejamento e implementação de linhas de cuidado numa dada região, é essencial o conhecimento da realidade local, condição igualmente necessária para a oferta racional de serviços de saúde a uma população específica, como a privada de liberdade no sistema prisional. Neste contexto, dentre os principais agravos em saúde observados estão as doenças respiratórias, como sinusite, rinite alérgica e bronquite crônica (MINAYO; RIBEIRO, 2016), e infectocontagiosas, como HIV/Aids, tuberculose, sífilis, hepatites B e C e outras de transmissão sexual (GOIS et al., 2012; VALENÇA et al., 2016). Além disso, manifestam-se os danos à saúde mental, decorrentes do estresse, da ansiedade, da depressão (SANTOS et al., 2016; CONSTANTINO; ASSIS; PINTO, 2016; MINAYO; RIBEIRO, 2016) e do uso problemático de drogas (MICHEL, 2016). Porém, os agravos psicossociais decorrentes do confinamento apresentam claras distinçôes de gênero, com maior prevalência de transtornos mentais graves entre as mulheres, em comparação com os presos do sexo masculino (SANTOS et al., 2016; MINAYO; RIBEIRO, 2016).

Portanto, compreender o encarceramento feminino é entender que as mulheres em situação de prisão constituem um grupo especialmente vulnerável, fruto das múltiplas vitimizaçóes sofridas durante sua trajetória vital e, muitas vezes, expostas a processos de revitimização a partir da violência institucional vivenciadas no ambiente carcerário. Nesse contexto, a invisibilidade das especificidades de gênero em prisão favorece a revitimização feminina, agora por parte da instituição total que as abriga (SCHULTZ, 2018). Logo, conhecer o perfil epidemiológico dessa população, monitorar as condiçóes de saúde e avaliar as ações ofertadas pelos serviços que se destinam a atendê-la é imprescindível, considerando a necessidade de qualificar e assegurar os fluxos assistenciais, a responsabilização e a resolutividade da assistência.

O princípio da integralidade como orientador do processo de trabalho das EABp, além de ser um eixo orientador do SUS, é também uma atitude de cuidado. Para alcançar a integralidade da atenção à saúde, é fundamental articular três elementos-chave: o acesso, a responsabilização e a autonomia (MEDEIROS, 2015). A condição do cuidado integral está associada à qualidade do acesso à saúde; está diretamente relacionado ao princípio da universalidade como direito de todo cidadão e dever do Estado. 
A responsabilização está fortemente relacionada ao estabelecimento de vínculo e à resolutividade da atenção. Nela expressam-se os elementos éticos, técnicos e políticos do ato de cuidar da saúde por parte das equipes. Por fim, mas não menos importante, está o produto do cuidado, ou seja, a autonomia do usuário. Isso significa que todo ato em saúde procurará respeitar a singularidade e a cidadania daquele que é cuidado, evitando tutela ou reificação do sujeito que busca atenção a sua saúde (MEDEIROS, 2015).

Em linhas gerais, a integralidade é passível de ser entendida de formas distintas (MATTOS, 2009), embora sempre indicando, em quaisquer delas, um rompimento do reducionismo e da objetivação dos sujeitos, presentes no cuidado à saúde e na prática da medicina ainda vigente. Pode, dessa maneira, representar a ampliação da coordenação do cuidado e da responsabilidade setorial e interprofissional.

Essa nova perspectiva de organização e gestão do trabalho em saúde aponta igualmente para novas pactuaçóes e orientaçóes na prática técnico-assistencial e político-organizacional, no âmbito da APS no sistema prisional. Pelo exposto, o presente trabalho busca identificar, através da perspectiva de uma EABp, quais limites e desafios para inclusão da população prisional feminina e egressa do sistema prisional nas Redes de Atenção à Saúde (RAS).

\section{Percurso metodológico}

Atualmente, o Sistema Prisional do Rio Grande do Sul (RS) conta com um total de 102 unidades prisionais, sendo cinco exclusivamente femininos. A pesquisa foi realizada com uma EABp implantada em um presídio feminino. Participaram dez profissionais de saúde provenientes de diferentes núcleos de formação: quatro da enfermagem (dois de nível superior e dois de nível técnico), três da medicina (clínico geral, psiquiatra e ginecologista), um do serviço social, um da psicologia e um da odontologia. A amostra foi por conveniência.

Os dados foram coletados em janeiro e fevereiro de 2015. Foram realizadas duas sessôes de grupo focal com a equipe de saúde em seu ambiente cotidiano de trabalho, ou seja, nas dependências da Unidade Básica de Saúde prisional (UBSp). Esta estratégia objetivou o diálogo em profundidade e com um nível maior de trocas entre os participantes da pesquisa. A interação dos participantes entre si e com a equipe de pesquisa conduziu a um contexto multidimensional, permitindo também 
uma abertura para compreender o modo como os fatores estruturais, de gestáo e de comunicação naquele contexto de trabalho poderiam impulsionar as conversas. Os pesquisadores procuraram respeitar as discussôes, confrontos e discordâncias - no caso, sobre o objeto em estudo, o acesso da população feminina privada de liberdade e egressas do sistema prisional nas Redes de Atenção à Saúde.

O material foi transcrito, salvo em documentos de texto e importado para o software QSR NVivo ${ }^{\oplus}$ versão 11 para Windows. O corpus foi analisado por meio do método da análise temática abdutiva, seguindo seis fases: familiarização com os dados, geração de códigos iniciais, busca pelos temas, revisão dos temas, definição dos temas e relatório/análise (BRAUN; CLARK, 2006).

$\mathrm{O}$ estudo resulta da dissertação de mestrado da primeira autora e seguiu os preceitos da Resolução do Conselho Nacional de Saúde no. 466/2012, que orienta pesquisas com seres humanos, tendo recebido parecer consubstanciado pelo Comitê de Ética em Pesquisa da Escola de Saúde Pública do Estado do Rio Grande do Sul, sob o CAAE 40678115.1.0000.5312, e pela Comissão de Pesquisa do Instituto de Psicologia da Universidade Federal do Rio Grande do Sul. O anonimato dos participantes foi preservado, e os excertos que serão apresentados neste trabalho foram codificados da seguinte forma: P1 (participante 1) até P10 (participante 10).

\section{Resultados e Discussão}

A análise da dimensão teórico-metodológica do estudo foi de caráter social construtivista e resultou em dois eixos temáticos: (a) Barreiras para inclusão das reclusas na RAS; e (b) Barreiras para inclusão das egressas na RAS, os quais serão apresentados na sequência.

\section{Barreiras para inclusão das reclusas na RAS}

A partir de pactuaçóes realizadas entre a Secretaria Estadual da Saúde (SESRS), a Superintendência dos Serviços Penitenciários (SUSEPE) do RS e a Secretaria Municipal de Saúde de Porto Alegre, além dos cuidados primários à saúde, é garantido às mulheres privadas de liberdade o acesso à assistência hospitalar e ambulatorial de serviços de apoio, diagnóstico e terapêutico na rede SUS local e regional.

São três os hospitais gerais de referência para a realização desses serviços: a Associação Hospitalar Vila Nova (clínica médica e dependência química/ desintoxicação); o Hospital Nossa Senhora da Conceição (alta complexidade) e o 
Hospital Presidente Vargas (gravidez de alto risco e parto). As pactuaçóes entre as três esferas estabelecem que a regulação e o gerenciamento de leitos, bem como a logística para viabilizar o transporte (acesso) das reclusas à unidade hospitalar estão sob a gestão da SUSEPE (RIO GRANDE DO SUL, 2018).

O fluxo assistencial para o atendimento das reclusas no âmbito especializado e de atenção a urgências e emergências da rede SUS municipal e regional é regulado, uma vez que a UBSp (como qualquer outro serviço de APS) não tem nem estrutura física e nem densidade tecnológica para exercer a atenção especializada. No entanto, os profissionais da EABp referiram uma lacuna entre o que é normatizado e o que realmente é possível ser acessado. Constatou-se que a inclusão das reclusas nos serviços de Atenção Secundária e Terciária da rede SUS municipal ocorria de maneira burocratizada e parcial. A dificuldade encontrada referia-se às especialidades médicas e odontológicas, tanto para consultas quanto para a internação.

Quanto às consultas, um dos problemas era a disponibilidade de atendimento pelo sistema municipal, através de agendamento e espera. Observa-se que o tempo de espera para acesso a uma consulta poderia ser ampliado pelo fato de necessitar apoio do setor da segurança (escolta, em especial), por vezes sem efetivo disponível para realizar o transporte até a unidade hospitalar. Essa condição levava os profissionais a refazerem o esforço, obrigando a nova espera. Com efeito, aumentava o desgaste da equipe e, obviamente, a indignação da população assistida.

Diante disso, as açôes de maior nível de articulação entre a EABp e os serviços de saúde do município (consultas e exames) eram priorizadas para as mulheres que cumpririam um maior tempo de pena naquela unidade prisional. Isso se justificava pelo fato de algumas estarem de "passagem na prisão" (P4) e, ao sair, seja por motivo de progressão de regime ou por livramento, não terem possibilidade de acesso ao atendimento pré-agendado pela EABp. Logo, a espera para atendimento gerava a descontinuidade do cuidado e o acesso burocratizado ao serviço, fazendo a equipe orientar-se também pela situação jurídica/penal da população atendida. Reiterou um dos profissionais:

Bom, em primeiro lugar a gente tem que saber, tipo, quanto tempo ela vai ficar aqui. Tu não vai te mobilizar tanto, mobilizar tanta gente, se depois consegue marcar [consulta e exame], fazer, fazer, fazer, daí ela vai embora. [...] o juiz manda ela embora, a gente nem fica sabendo e ela sai daqui sem as consultas que tu marcou para ela, tirando a vaga de outra que está precisando. Então, uma das coisas é saber que tipo de... qual é a situação jurídica dela. A situação da pena dela; para ter uma orientação no atendimento (P4). 
Observa-se que um dos pontos de análise que não se mostraram destacados nas narrativas dos participantes da pesquisa, mas que se demonstra importante para a atenção à saúde nas prisóes é o fluxo necessário para a continuidade do acompanhamento em saúde, a partir do momento em que a reclusa é transferida para outra unidade prisional. Durante a realização do estudo, constatou-se que os sistemas de saúde e prisional do Rio Grande do Sul não dispunham de integração entre os seus sistemas de informação. Apesar dos avanços da PNAISP, estratégias efetivas que, por exemplo, interligam prontuários eletrônicos de diferentes cidades não foram concretizadas, trazendo implicações no acesso desta população às RAS.

Se por um lado havia barreiras para garantir o acesso às consultas especializadas, por outro, evidenciava-se um maior vazio assistencial no âmbito da Rede de Atenção Psicossocial (RAPS) e o cuidado em saúde mental. De acordo com os profissionais, as mulheres privadas de liberdade não acessavam a RAPS local ou regional. Informaram que o único serviço de referência para atenção às urgências em saúde mental era o Instituto Psiquiátrico Forense Doutor Maurício Cardoso (IPF), em Porto Alegre. Sendo este um hospital de custódia e tratamento psiquiátrico, criado para a internação e custódia de pessoas com transtorno mental em conflito coma lei.

Importa salientar que ainda não existem dispositivos ou pactuaçôes estaduais e municipais que inclua a população prisional do RS nos serviços da RAPS. Diante disso, cabe a cada unidade prisional e aos profissionais que compóem as EABp articular o fluxo assistencial diretamente com os serviços de saúde mental da rede. Em contraponto, os participantes da pesquisa afirmaram ter realizado, por diversas vezes, o contato com os serviços do Distrito Sanitário onde o presídio estava sediado, mas a justificativa para o não atendimento recaía na necessidade de realização de escolta.

Um profissional referiu que havia o despreparo da rede de saúde mental: "A rede ainda não está preparada para atender paciente psiquiátrico do sistema [prisional]" (P1). Tal despreparo pode ser ponderado a partir de dois ângulos. Primeiramente, pela necessidade de adaptar o serviço/atendimento extramuros às necessidades da Segurança (algemas, vigilância, armamento de fogo e outros), as quais são estranhas e alheias à realidade da maioria dos serviços e dos profissionais de saúde. E, também, pela negligência da rede SUS municipal em assegurar que a APS prisional seja reconhecida como um ponto estratégico da RAS e, verdadeiramente, assuma a coordenação do cuidado da usuária na rede. 
A coordenação do cuidado enquanto atributo da APS existia mais como uma prática discursiva associada aos parâmetros normativos do que como uma verdadeira orientação para o trabalho da EABp. O modelo de atenção preconizado nos atos normativos era distante do cuidado possível de ser ofertado dentro do presídio ou fora dele. Desse modo, é possível compreender o motivo pelo qual os trabalhadores da EABp afirmaram que "essa lei [Reforma Psiquiátrica], para nós, não serve. A gente está no século passado" (P5) e que a atenção à saúde na prisão pode ganhar status de cuidados paliativos “[...] que a gente tem que saber que são paliativos [...]" (P4).

Por mais que, hoje, a normativa é tu encaminhar para o CAPS [Centro de Atenção Psicossocial] e não mais para o IPF [Instituto Psiquiátrico Forense Doutor Maurício Cardoso, em Porto Alegre], é isso que deveria prevalecer. Faz mais de dez anos, tu não tem suporte para poder encaminhar ela (P3).

Se ela precisar de uma internação, tu não tem como fazer escolta num hospital, na urgência psiquiátrica. Às vezes, o hospital nem quer escolta. E tem que ter escolta. Então, a gente continua ainda recorrendo ao IPF, que lá ela vai e fica porque é a SUSEPE (P2).

Outra situação explorada diz respeito às dificuldades para a realização do prénatal gestantes com quadro agudo de sofrimento psíquico, decorrente de uso, abuso e intoxicação por substâncias psicoativas. Evidencia, assim, a violência institucional que as reclusas são submetidas, sobretudo através da negligência e da omissão por parte do poder público, como se observa no excerto:

Nós tivemos três casos, por exemplo, de mães gestantes com problema psiquiátrico e uso de drogas e nós não tínhamos o que fazer. Ela precisando, já não estava resolvendo o atendimento de ambulatório que estava oferecendo para ela aqui [no presídio] e ela já tinha uma internação [psiquiátrica]. Onde levar ela, entende? O [suprimido o nome da instituição hospitalar] não aceita gestante [...]. Já o hospital [suprimido o nome da instituição] não aceitava porque tinha que ter escolta lá dentro, os psiquiatras, eles não aceitam [...]. E nós ficamos num bico de sinuca, e nós abraçamos ela da melhor maneira possível, sem internação, entende? Mas precisando de uma internação. Então, nós não temos esse suporte. Nós não temos (P1).

A par dessa realidade, constatou-se que, no período pesquisado, houve grave violação do direito constitucional à saúde da mulher gestante, ampliando as iniquidades e, consequentemente, a vulnerabilidade desta população. Além disso, é possível observar que os profissionais da EABp eram por vezes pressionados, por variadas fontes, a dar respostas para além de suas competências técnicas e legais, ficando expostos a situaçóes dilemáticas diante dos princípios éticos profissionais e do compromisso de prover cuidado para amenizar sofrimentos. Por esse motivo, a 
atuação da EABp oscilava entre o cuidado necessário e o possível, recaindo, muitas vezes, na resolutividade forçada (BARSAGLINI; KEHRIG; ARRUDA, 2015).

Esses dados evidenciam as dificuldades em gerir a garantia do acesso à atenção secundária especializada e, principalmente, à atenção terciária, no âmbito da Saúde Mental, pois os dispositivos de integraçáo, no momento do estudo, existentes, não seriam suficientes para assegurar um sistema de referência em pleno funcionamento. Isso leva a inferir que a EABp tem uma média integração com os serviços da RAS municipal, supondo a necessidade de investimentos para reconhecer as especificidades desse serviço na rede de atenção. A questão transforma-se, portanto, em um desafio significativo para a integração ou criação de dispositivos entre a APS prisional e a rede psicossocial, bem como para o alcance dos princípios da Reforma Psiquiátrica no âmbito do sistema prisional gaúcho.

\section{Barreiras para inclusão das egressas na RAS}

Além das barreiras para garantir o acesso das reclusas na rede SUS do município, constatou-se que a EABp também apresentava dificuldades para coordenar o cuidado no momento de transição da mulher egressa do sistema prisional para os serviços de saúde de referência distrital e comunitária. Na perspectiva de um dos profissionais, esse problema estaria associado, novamente, à burocracia para acessar os serviços primários territoriais, dificultando a articulação entre tais serviços e a EABp:

[...] a única forma de acesso é por fichas distribuídas. Tem que ir lá de manhã, pegar
uma ficha e agendar [...] existe um intervalo muito grande entre ela sair e conseguir ter
essa entrada na Unidade [ESF ou UBS], é um intervalo grande. Aí nesse meio já se perde
várias coisas (P2).

A dificuldade de acesso é um elemento importante e constitui um obstáculo à integralidade da atenção. Porém, por trás dessa prerrogativa, percebeu-se, quando da pesquisa, a insuficiência de mecanismos de diálogo e comunicação dos profissionais da EABp com os demais serviços da rede. Nesse caso, a dificuldade em coordenar o cuidado para fora dos muros do presídio pode ser em decorrência, sobretudo, do desconhecimento de tecnologias de microgestáo da clínica. Consequentemente, da não utilização de tecnologias que poderiam contribuir com essa comunicação e integração.

Instrumentos como a Gestão de Caso e o Projeto Terapêutico Singular, ou até mesmo o acionamento de serviços de apoio matricial, poderiam colaborar com o processo de transição da APS da prisão para os serviços de saúde do território 
de proveniência da egressa, porém essa prática ainda não fazia parte da prática daquela equipe. Esse dado aponta que o desconhecimento de ferramentas de gestão do cuidado, ao lado da falta de planejamento do processo de trabalho, afetou a coordenação do cuidado e a descontinuidade do tratamento fora da prisão.

Embora a elaboração de planos de cuidado não fizesse parte do trabalho da $\mathrm{EABp}$, havia uma organização para que o tratamento das mulheres diagnosticadas com HIV/Aids fosse continuado nos serviços especializados da RAS. Antes de saírem da prisão, passavam por uma consulta médica na UBSp. As consultas com especialistas (infectologista) eram pré-agendadas. As mulheres eram orientadas pela EABp a continuar o tratamento e recebiam cópia dos documentos do prontuário de saúde, enquanto os medicamentos (antirretrovirais) também eram fornecidos por um período suficiente até que a consulta especializada ocorresse:

Normalmente, a gente avisa: ó, se caso você [reclusa] for embora [da prisão] depois das $17 \mathrm{~h}$, no outro dia pede para o teu familiar vir aqui. A gente dá xerox de todos os teus encaminhamentos, exames, e se tu for até as $17 \mathrm{~h}$, bate aqui que a gente te dá. A gente dá essa orientação a semana inteira. E elas realmente vêm, se não vem ela, vem o familiar, é super tranquilo (P3).

[...] quando é um HIV, né, por exemplo, já sai com uma medicação com o tempo adequado para ela poder se manter até ela se introduzir sozinha. Algumas pessoas têm recurso e algumas não têm recurso (P1).

Esses excertos evidenciam um trabalho importanteenecessário, porém insuficiente, pois focalista. De tal maneira, legitima-se a ideia de que a responsabilidade pela continuação do tratamento é, única e exclusivamente, da usuária, não importando se ela possui (ou não) recursos para tal (passagem de ônibus, condições físicas e psíquicas, acompanhante/família etc.). Essa situação tornou-se ainda mais grave ao constatar-se que as mulheres com diagnóstico psiquiátrico saíam do Sistema Prisional sem encaminhamento ou pré-agendamento de consulta em serviços especializados da RAPS. Também saíam sem a prescrição de psicotrópicos. Importa lembrar que, na ocasião da pesquisa, os profissionais estimavam que 50\% das recusas apresentavam alguma morbidade psiquiátrica e realizavam tratamento farmacológico.

As consequências negativas estão associadas à inexistência de um plano de cuidado para o processo da saída do cárcere: a usuária pode ficar sem medicamento até conseguir acessar o serviço de referência territorial; pode ter "recaída" (P9) ou fazer uso abusivo de drogas; e, não menos importante, reincidir e sofrer novo processo de reencarceramento, estimulando o fenômeno de "porta giratória" nas 
prisôes (FRAZIER et al., 2015). Identificam-se tais situações no subsequente conjunto de excertos:

A [suprimido o nome da reclusa] é um caso que retornou agora [reincidência]. Ela contou
que ela saiu [da prisão] e voltou e aí ela disse que não teve acompanhamento nenhum na
rede. Nem na assistência [social] e nem saúde (P9).
[...] essas que são dependentes químicas, por exemplo, acho que elas deveriam passar
por uma consulta com um psiquiatra antes de sair [da prisão], para já sair com a receita
[prescriçáo médica], porque, para não ter período sem tratamento. E daí já facilita a
recaída, né (P7).

Um estudo internacional constatou que a transição do atendimento nos serviços de APS da prisão para os serviços territoriais é realizada de maneira insuficiente, inadequada ou inexistente (BINSWANGER et al., 2011). Há indícios de que um dos fatores para a dificuldade de acesso dos egressos aos serviços de Atenção Primária e Secundária da comunidade é decorrente da falta de planejamento dos serviços de APS da prisão, que permite que casos complexos sejam subvalorizados no processo de livramento. Além disso, estes são frequentemente liberados com uma oferta muito limitada de medicamentos (BINSWANGER et al., 2011; KINNER et al., 2016; LEUTWYLER; HUBBARD; ZAHND, 2017). Tais dados coincidem com as evidências qualitativas discutidas no presente estudo.

A descoordenação do cuidado aumenta a vulnerabilidade e os riscos de náo continuidade do tratamento, bem como agrava as condições de saúde da população egressa. Consequentemente, aumentam as probabilidades de suicídio, hospitalização e reencarceramento (LEUTWYLER; HUBBARD; ZAHND, 2017), as quais poderiam ser minimizadas ou até mesmo evitadas.

Outros estudos evidenciaram que o primeiro mês de pós-reclusão (LARNEY et al., 2015) eleva o risco de mortes por causas externas como suicídio, overdose ou intoxicação por drogas e homicídio. Essa probabilidade aumenta quando se trata de pessoas com problemas de saúde decorrentes do uso de substâncias (LARNEY et. al., 2015; CHANG et al., 2015); com histórico prévio de reencarceramento (LARNEY et. al., 2015); com baixa rede de apoio (familiar, de serviços públicos e comunitários) (CHANG et al., 2015) e quando se trata de gestantes ou em situação de pós-parto (ZLODRE; FAZEL, 2012).

Esses dados indicam que a experiência de reclusão é um fator mortificante, podendo ser acentuada pela falta de coordenação do cuidado e implementação de 
ferramentas para realizar a Gestão de Caso, em especial para os egressos com condiçóes complexas de saúde (alto risco e vulneráveis) (CHANG et al., 2015; LEUTWYLER; HUBBARD; ZAHND, 2017). A literatura nacional especializada em APS reitera que a Gestão de Caso é um dos componentes da gestão da clínica. É considerada como o instrumento mais adequado para os profissionais e usuários dos serviços de cuidados primários, pois mantém o foco no acompanhamento de condiçóes crônicas complexas que necessitam de um planejamento ampliado (MENDES, 2015), ou seja, colaborativo (trabalho em equipe) e integrado (com os demais serviços da rede).

É crucial o oferecimento pelos serviços de programas ou de planos individuais de saída da prisão, ou seja, que auxiliem as EABp, sobretudo no processo de transição da APS prisional para os serviços da RAS de referência comunitária. A implementação de planos de cuidado - seja por Gestão de Caso, seja por PTS - é fundamental, pois esses instrumentos favorecem o acompanhamento e o fortalecimento da rede de apoio dessa população, especialmente dos indivíduos que apresentam condiçóes complexas de saúde (BINSWANGER et al., 2011; KINNER et al., 2016; LEUTWYLER; HUBBARD; ZAHND, 2017).

Soma-se a isso a necessidade de implementar programas para treinamento adequado (BINSWANGER et al., 2011; KINNER et al., 2016; LEUTWYLER; HUBBARD; ZAHND, 2017) e de educação permanente aos profissionais da EABp, pois tais lacunas acabam por trazer consequências negativas para a saúde das reclusas e egressas do sistema prisional, condição que se irradia para as famílias e para a comunidade como um todo.

Invariavelmente, há necessidade de esforço mútuo entre os profissionais da saúde, da Administração Penitenciária, da Gestão da Saúde e da Justiça Criminal. Do contrário, corre-se o risco de reproduzir práticas voltadas unicamente para o desencarceramento (liberação do sistema), e não para a desinstitucionalização. Em face disso, a desinstitucionalização deve se tornar uma premissa para o trabalho em saúde no sistema prisional.

\section{Considerações finais}

As evidências do estudo qualitativo apontaram que, apesar de a APS prisional já ser considerada, por meio das orientaçóes normativas, a principal porta de entrada da populaçáo prisional no sistema de saúde, ainda precisa ser organizada para se 
tornar ordenadora no acesso à rede. A atenção especializada também necessita ser qualificada com a ampliação e otimização da oferta de serviços mais equitativos e integrais, de acordo com o modelo de cuidado em rede. Tem-se como desafio a construção de uma rede capaz de coordenar de forma qualificada e integrada a gestão dos fluxos assistenciais para a atenção às mulheres privadas de liberdade usuárias do sistema de saúde.

As dificuldades para a inserção e referenciamento das egressas nos serviços de saúde e socioassistenciais, contribui - em alguma medida - para a descontinuidade do tratamento em saúde e para o fenômeno de porta giratória, haja vista a prevalência do reencarceramento e da falta de acompanhamento da população egressa pelas políticas sociais públicas e comunitárias.

Tais barreiras estão associadas a fatores de macrogestão da clínica como a racionalidade burocrática do SUS (barreiras para o acesso aos serviços de cuidados primários); e de microgestão da clínica como a pouca comunicação e articulação da EABp com os demais serviços da rede SUS, de modo a favorecer a descoordenação do cuidado e a desresponsabilização entre os serviços. Além disso, a pouca integração entre EABp e os demais profissionais da segurança pública podem dificultar a implementação de fluxos conjuntos e planos de cuidado integrados. Não menos importante, o desconhecimento de tecnologias de microgestão como a implementação da Gestão de caso e do PTS, com vistas à produção do cuidado compartilhado e integrado e de planos individuais de saída da prisão.

Sem essa prerrogativa de gestão, corre-se o risco de limitar a atenção à saúde no sistema prisional à fase inicial de elaboração de normas, sem que se garantam as condiçôes para que possam ser cumpridas: ou por não dispor dos recursos necessários ou, principalmente, por não contar com a adesão e o compromisso dos que deveriam cumpri-las.

Esse contexto se agrava quando percebemos que as políticas e os serviços executados em âmbito prisional foram desenhados - historicamente - para a população masculina. As mulheres reclusas possuem necessidades específicas, que não podem ser obviadas pela equipe de saúde e demais profissionais que atuam dentro dos estabelecimentos prisionais. Destacamos, pois, a necessidade de implementar esforços mútuos entre os profissionais da saúde, da Administração Penitenciária, da Gestão da Saúde e da Justiça Criminal com foco na desinstitucionalização e não apenas para o desencarceramento feminino. Trata-se de uma luta em defesa da vida, 
pela garantia dos direitos humanos e pela dignidade da saúde das mulheres que estão ou estiveram subordinadas a esse Sistema. ${ }^{1}$

\section{Agradecimentos}

O estudo foi financiado pela FAPERGS, sob o Edital "Programa Pesquisa para o SUS (PPSUS), vigente de 2013 a 2015.

\section{Referências}

BRASIL. Conselho Nacional de Justiça. Regras de Bangkok - Regras das Naçôes Unidas para $o$ tratamento de mulheres presas e medidas náo privativas de liberdade para mulheres infratoras. Traduzidas para o português, 2016. Disponível em: <https://www.cnj.jus.br/wp-content/ uploads/2019/09/cd8bc11ffdcbc397c32eecdc40afbb74.pdf>. Acesso em: 12 dez. 2019.

. Portaria GM/MS no 01, de 02 de janeiro de abril de 2014. Institui a Política Nacional de Atenção Integral à Saúde das Pessoas Privadas de Liberdade no Sistema Prisional (PNAISP), no âmbito do Sistema Único de Saúde (SUS). Brasília (DF), 2014a. Disponível em: <http://bvsms. saude.gov.br/bvs/saudelegis/gm/2014/pri0001_02_01_2014.html>. Acesso em: 12 dez. 2019.

. Portaria GM/MS no 482 de $1^{\circ}$ de abril de 2014. Institui a Política Nacional de Atenção Integral à Saúde das Pessoas Privadas de Liberdade no Sistema Prisional (PNAISP) no âmbito do Sistema Único de Saúde (SUS). Brasília (DF), 2014b. Disponível em: <http://bvsms.saude.gov. br/bvs/saudelegis/gm/2014/prt0482_01_04_2014.htm>. Acesso em: 12 dez. 2019.

BARSAGLINI, R. A.; KEHRIG, R. T.; ARRUDA, M. B. de. Análise da percepção de gestores sobre a gestão da política de saúde penitenciária em Mato Grosso, Brasil. Saúde e Sociedade, v. 24, n. 4, p. 1119-1136, 2015. Disponível em: < https://www.scielosp.org/scielo.php?pid=S010412902015000401119\&script=sci_abstract\&tlng=pt>. Acesso em: 12 dez. 2019.

BINSWANGER, I. A. et al. "From the prison door right to the sidewalk, everything went downhill," A qualitative study of the health experiences of recently released inmates. International journal of law and psychiatry, v. 34, n. 4, p. 249-255, 2011. Disponível em: <https://www. sciencedirect.com/science/article/pii/S0160252711000628>. Acesso em: $12 \mathrm{dez} .2019$.

BRAUN, V.; CLARKE, V. Using thematic analysis in psychology. Qualitative research in psychology, v. 3, n. 2, p. 77-101, 2006. Disponível em: <http://eprints.uwe.ac.uk/11735/2/ thematic_analysis_revised>. Acesso em: $12 \mathrm{dez} .2019$.

CHANG, Z. et al. Substance use disorders, psychiatric disorders, and mortality after release from prison: a nationwide longitudinal cohort study. The Lancet Psychiatry, v. 2, n. 5, p. 422-430, 2015. Disponível em: <https://www.sciencedirect.com/science/article/pii/ S2215036615000887>. Acesso em: 12 dez. 2019. 
CONSTANTINO, P.; ASSIS, S. G. de; PINTO, L. W. O impacto da prisão na saúde mental dos presos do estado do Rio de Janeiro, Brasil. Ciência \& Saúde Coletiva, v. 21, p. 2089-2100, 2016. Disponível em: <https://www.scielosp.org/article/csc/2016.v21n7/2089-2100/pt/>. Acesso em: 1 jun. 2020.

FRANCO, T. B.; MAGALHÂES JÚNIOR, H. M. Integralidade na assistência à saúde: a organização das linhas do cuidado. $O$ trabalho em saúde: olhando e experienciando o SUS no cotidiano. São Paulo: Hucitec, 2003. p. 125-34.

FRAZIER, B. D. et al. The impact of prison deinstitutionalization on community treatment services. Health \& justice, v. 3, n. 1, p. 9, 2015. Disponível em: <https://healthandjusticejournal. biomedcentral.com/articles/10.1186/s40352-015-0021-7>. Acesso em: 12 dez. 2019.

GOIS, S. M. et al. Para além das grades e puniçóes: uma revisão sistemática sobre a saúde penitenciária. Ciência \& Saúde Coletiva, v. 17, n. 5, p. 1235-1246, 2012. Disponível em: <http://www.producao.usp.br/bitstream/handle/BDPI/39693/S1413- 81232012000500017. pdf?sequence=1>. Acesso em: 01 jun. 2020.

KINNER, S. A. et al. Low-intensity case management increases contact with primary care in recently released prisoners: a single-blinded, multisite, randomised controlled trial. Journal of Epidemiology \& Community Health, v. 70, n. 7, p. 683-688, 2016. Disponível em: <https:// www.ncbi.nlm.nih.gov/pubmed/26787201>. Acesso em: 12 dez. 2019.

LARNEY, S. et al. Variation in mortality risk of people released from prison. The Lancet Psychiatry, v. 2, n. 8, p. 681, 2015. Disponível em: <http://www.thelancet.com/pdfs/journals/ lanpsy/PIIS2215-0366(15)00235-7.pdf>. Acesso em: 12 dez. 2019.

LEUTWYLER, H.; HUBBARD, E.; ZAHND, E. Case management helps prevent criminal justice recidivism for people with serious mental illness. International journal of prisoner health, v. 13, n. 3/4, p. 168-172, 2017. Disponível em: <https://www.ncbi.nlm.nih.gov/ pubmed/28914124>. Acesso em: $12 \mathrm{dez} .2019$.

MATTOS, R. A. Os sentidos da integralidade: algumas reflexóes acerca de valores que merecem ser defendidos. In: PINHEIRO, R.; MATTOS, R. A. (Org). Os sentidos da integralidade na atenção e no cuidado à saúde. 8. Ed. Rio de Janeiro: ABRASCO, 2009. p. 43-68.

McDONALD, K. et al. Care Coordination Atlas Version. Rockville: AHRQ Publication; 2014. Disponível em: <https://www.ahrq.gov/sites/default/files/publications/files/ccm_atlas.pdf>. Acesso em: 12 dez. 2019.

MEDEIROS, R. H. A. de. Uma noçấo de matriciamento que merece ser resgatada para o encontro colaborativo entre equipes de saúde e serviços no SUS. Physis: Revista de Saúde Coletiva, v. 25, p. 1165-1184, 2015. Disponível em: <https://www.scielosp.org/article/physis/2015. v25n4/1165-1184/pt/>. Acesso em: 12 dez. 2019. 
MENDES, E. V. A construção social da atenção primária à saúde. Brasilia: Conselho Nacional de Secretários de Saúde, 2015. Disponível em: <http://www.saude.go.gov.br/wp-content/ uploads/2017/07/a-construcao-social-da-aps-mendes-2015-1.pdf>. Acesso em: 12 dez. 2019.

MICHEL, L. Drug use in prisons: strategies for harm reduction (ANRS-PRIDE Program). Ciênc. saúde coletiva, Rio de Janeiro, v. 21, n. 7, p. 2081- 2088, July 2016. Disponível em: <http:// www.scielo.br/pdf/csc/v21n7/1413-8123-csc-21-07-2081.pdf>. Acesso em: 01 jun. 2020.

MINAYO, M. C. de S.; RIBEIRO, A. P. Condiçôes de saúde dos presos do estado do Rio de Janeiro, Brasil. Ciência \& Saúde Coletiva, v. 21, n. 7, 2016. Disponível em: <http://www.scielo. br/pdf/csc/v21n7/1413-8123-csc-21-07-2031.pdf>. Acesso em: 01 jun. 2020.

RIO GRANDE DO SUL. Secretaria da Saúde. Resolução no 099/18 - CIB/RS. Porto Alegre: Secretaria Estadual da Saúde, 2018.

SANTOS, M. M. et al. Transtorno Mental e Prisão. In: TABORDA, J. G. V.; ABDALLA FILHO, E.; CHAUB, M. (Orgs.). Psiquiatria Forense. 3ª. Ed. Porto Alegre: Artmed, 2016.

SCHULTZ, A. L. V. et al. Saúde no Sistema Prisional: um estudo sobre a legislaçáo brasileira. Argumentum, v. 9, n. 2, p. 92-107, 2017. Disponível em: <https:/www.redalyc.org/ pdf/4755/475555301009.pdf>. Acesso em: 12 dez. 2019.

SCHULTZ, A. L. V. O apoio matricial como metodologia para o trabalho em saúde no sistema prisional: fatores favoráveis, desfavoráveis e contribuições. 2018. Dissertação (Mestrado em Política Social e Serviço Social) - Programa de Pós-Graduação em Política Social e Serviço Social, Universidade Federal do Rio Grande do Sul, Porto Alegre, 2018. Disponível em: <https:// lume.ufrgs.br/bitstream/handle/10183/182009/001076288.pdf?sequence=1\&isAllowed $=y>$. Acesso em: 01 jun. 2020.

UNODOC. Regras Minimas das Naçóes Unidas para o Tratamento de Reclusos (Regras de Nelson Mandela), 2015. Disponível em: <https://www.unodc.org/documents/justice-and-prisonreform/Nelson_Mandela_Rules-P-ebook.pdf>. Acesso em: 12 dez. 2019.

VALENÇA, M. S. et al. Tuberculose em presídios brasileiros: uma revisão integrativa da literatura. Ciênc. saúde coletiva, Rio de Janeiro, v. 21, n. 7, p. 2147-2160, July 2016. Disponível em: <http://www.scielo.br/scielo.php?pid=S1413-81232016000702147\&amp;script=sci_ arttext\&amp;tlng=en\&gt>. Acesso em: 01 jun. 2020.

ZLODRE, J.; FAZEL, S. All-cause and external mortality in released prisoners: systematic review and meta-analysis. American Journal of Public Health, v. 102, n. 12, p. e67-e75, 2012. Disponível em: <https://www.ncbi.nlm.nih.gov/pmc/articles/PMC3519300/>. Acesso em: 12 dez. 2019.

WORLD HEALTH ORGANIZATION (WHO). Health in prisons: a WHO guide to the essentials in prison health. Copenhagen: Organization Regional Office for Europe, 2007. Disponível em: <http://www.euro.who.int/_data/assets/pdf_file/0009/99018/E90174.pdf>. Acesso em: 12 dez. 2019. 


\section{Nota}

${ }^{1}$ A. L. V. Schultz: participou da concepção do artigo, redação, análise e aprovou a versão final a ser publicada. R. M. Dotta e B. S. Stock participaram da concepção do artigo, redação, revisão crítica relevante do conteúdo intelectual e aprovou a versão final a ser publicada. M. T. G. Dias participou da concepção do artigo, realizou a revisão crítica relevante do conteúdo intelectual e aprovou a versão final a ser publicada. 


\section{Abstract}

Limits and challenges for women deprived of their liberty and those released from the prison system in the Health Care Networks

The work analyzes the limits and challenges for the access of the female prison population released from the prison system in the services of the Health Care Network (RAS). This is a qualitative, exploratory and descriptive study conducted with ten workers from a prison primary care team in a female prison in the South Region of Brazil. Focus group sessions were held and the data were analyzed thematically, resulting in two axes: (a) Barriers for the inclusion of prisoners in the RAS; and (b) Barriers to the inclusion of graduates in the RAS. Even though the basic prison health unit is considered the main gateway for the prison population to the health system, it still needs to be organized to become the ordering of women's health care. Difficulties in accessing these women in extramural health services contribute to the discontinuity of treatment, given the lack of monitoring of this population by the components of the HCN, especially by mental health services. It is necessary to advance in the expansion and optimization of more equitable and comprehensive services, stimulating the integration in the flow management between the primary prison care and the other RAS devices.

Keywords: prisons; Primary Health Care; Health Care Networks; mental health; gender and health. 